\title{
UMA METODOLOGIA GERAL PARA CORRIGIR DISTRIBUIÇÕES DE ESTATÍSTICAS
}

\author{
Gauss MI. Cordeiro* \\ Silvia L. P. Ferrari**
}

\section{Resumo}

O objetivo deste artigo é mostrar que qualquer estatística-teste, satisfazendo condições gerais de regularidade, pode ser ajustada de modo que sua distribuição até ordem $O\left(n^{-1}\right)$ concorde com uma distribuição arbitrária de primeira ordem, onde $n$ é o tamanho da amostra. Este artigo estende o trabalho de Cordeiro e Ferrari (1991) para vários outros testes importantes em Econometria.

\section{Abstract}

The object of this paper is to show that for any test statistic satisfying fairly general regularity conditions, we can easily construct an adjusted statistic which has the same distribuition to order $O\left(n^{-1}\right)$ of an arbitrary first-order approximating distribution, where $n$ is the sample size. This paper extends the result of the closely related paper by Cordeiro and Ferrari (1991) to cope with several other important tests in Econometrics.

Palavras-Chave: Correção de Bartlett, cumulantes, distribuição de referência, estatística escore, estimativa de máxima verossimilhança, expansão assintótica

Código JEL: C12

* Departamento de Estatística, Universidade Federal de Pernambuco, Cidade Universitária, 50740-540, Recife, PE.

** Departarmento de Estatística, Instituto de Matemática e Estatistica, Universidade de São Paulo, 05389-970, S. Paulo, SP.

R. de Econometria Rio de Janeiro v. 15, $\mathrm{n}^{\mathrm{0}}$ 2, pp.1-30 Novembro/Março 1996 
Metodologia para corrigir distribuiçōes de estatísticas

\section{Introdução.}

Neste artigo, apresentamos uma metodologia geral para corrigir qualquer estatística-teste com distribuição de referência arbitrária que é usada como primeira aproximação para a distribuição da estatística. Esta metodologia generaliza o resultado de Cordeiro e Ferrari (1991) para qualquer combinação estatística - distribuição de referência. Demonstramos a existência de uma estatística corrigida cuja distribuição é idêntica àquela de referência até ordem $n^{-1}$, onde $n$ é o tamanho da amostra. A estatística corrigida é função de derivadas da distribuição de referência e dos cumulantes da estatística original e da variável de referência. Dividimos o artigo em 7 seções. Nesta seção, revisamos as expansões clássicas das funções geratrizes de momentos e cumulantes de uma variável aleatória. $\mathrm{Na}$ Seção 2, expressamos a função geratriz de momentos de uma estatística qualquer $S$ em termos da função geratriz de momentos de uma variável aproximada $Z$ e dos cumulantes de $S$ e $Z$. Expansões do tipo Edgeworth para a densidade e distribuição acumulada de uma variável aleatória contínua são revistas na Seção 3. Apresentamos, na Seção 4, uma fórmula geral para corrigir qualquer estatística-teste com distribuição de referência arbitrária. Esta fórmula é bastante útil em aplicações econométricas e estatísticas. Alguns casos especiais são analisados na Seção 5. Na Seção 6, apresentamos algumas estatísticas corrigidas de interesse prático, como por exemplo, a estimativa de máxima verossimilhança corrigida no caso uniparamétrico. Finalmente, na Seção 7, tecemos alguns comentários conclusivos.

Seja $S$ uma variável aleatória arbitrária que pode representar uma estatística qualquer. Sejam $K_{S}(t)$ e $M_{S}(t)$ as funções geratrizes de cumulantes e de momentos de $S$, respectivamente. Representamos por $\kappa_{1}, \kappa_{2}, \ldots$ e $\mu_{1}^{\prime}, \mu_{2}^{\prime}, \ldots$ os cumulantes e momentos ordinários de $S$ cujas ordens são $1,2, \ldots$, respectivamente. Sabemos que $M_{S}(t)=E\{\exp (t S)\}$, $I_{S}(t)=\log M_{S}(t)$ e que as $r$-ésimas derivadas de $K_{S}(t)$ e $M_{S}(t)$ no ponto $t=0$, supondo que estas derivadas existem, se igualam aos 
$r$-ésimos cumulante e momento de $S$, respectivamente.

A função geratriz de momentos (FGM) de $S$ é representada pela expansão

$$
M_{S}(t)=1+\mu_{1}^{\prime} t+\mu_{2}^{\prime} \frac{t^{2}}{2 !}+\cdots+\mu_{r}^{\prime} \frac{t^{r}}{r !}+\cdots,
$$

suposta convergente para todo $|t|$ suficientemente pequeno. A soma ilimitada em (1) pode ser divergente para todo real $|t|>0$, porque alguns dos momentos de ordem superior são infinitos ou porque os momentos, embora finitos, aumentam rapidamente forçando a divergência. Nestes casos, pode-se trabalhar com expansões finitas até um certo número de termos, especificando a ordem do erro como função do tamanho da amostra $n$ ou de alguma quantidade relacionada a $n$. Feller (1971, Seções VII.3, VII.6 e XV.4) apresenta condições que limitam a taxa de crescimento dos momentos de ordem impar.

A funçüo geratriz de cumulantes (FGC) é expandida como

$$
K_{S}(t)=\kappa_{1} t+\kappa_{2} \frac{t^{2}}{2 !}+\cdots+\kappa_{r} \frac{t^{r}}{r !}+\cdots
$$

Das equações (1) e (2), concluímos que

$$
\begin{aligned}
& \exp \left\{\kappa_{1} t+\kappa_{2} \frac{t^{2}}{2 !}+\cdots+\kappa_{r} \frac{t^{r}}{r !}+\cdots\right\} \\
& =1+\mu_{1}^{\prime} t+\mu_{2}^{\prime} \frac{t^{2}}{2 !}+\cdots+\mu_{r}^{\prime} \frac{t^{r}}{r !}+\cdots
\end{aligned}
$$

Expandindo em série de Taylor a função exponencial anterior e igualando os coeficientes de mesma potência em $t$, expressamos os momentos ordinários de $S$ em termos dos seus cumulantes de mesma ordem e de ordem inferior. As fórmulas dos cumulantes de $S$ em 
termos dos seus momentos ordinários podem ser obtidas facilmente expandindo o logaritmo abaixo em série de Taylor

$$
\kappa_{1} t+\cdots+\kappa_{r} \frac{t^{r}}{r !}+\cdots=\log \left\{1+\mu_{1}^{\prime} t+\cdots+\mu_{r}^{\prime} \frac{t^{r}}{r !}+\cdots\right\}
$$

e igualando os coeficientes de mesma potência em $t$.

Assim, existe uma relação biunívoca entre momentos e cumulantes. Entretanto, os cumulantes oferecem mais vantagens em termos estatísticos do que os momentos. Entre estas vantagens, citamse:

(a) um grande número de cálculos estatísticos usando cumulantes são mais fáceis do que os cálculos correspondentes através de momentos;

(b) para variáveis aleatórias independentes, os cumulantes de uma soma são, simplesmente, somas dos cumulantes das variáveis individuais;

(c) séries do tipo Edgeworth para aproximar densidades e distribuições (vide Seção 3) e logaritmos de densidades são expressos de forma mais conveniente via cumulantes ao invés de momentos;

(d) os cumulantes de inúmeras distribuições podem ter ordens préestabelecidas, o que não ocorre com os momentos. Por exemplo; os coeficientes de $I_{S}(t)$ em (2) são mais fáceis de serem limitados em potências de $n$ do que aqueles correspondentes em $\exp \left\{K_{S}(t)\right\}$

(e) considerando a aproximação normal (vide Seção 3), os cumulantes (mas não os momentos) de ordens superiores a um valor especificado podem, usualmente, ser ignorados, pois tendem a zero mais rapidamente que os demais quando o tamanho da amostra cresce. 
Além destas vantagens, os cumulantes têm interpretação simples. Os primeiro e segundo cumulantes são o valor médio e a variância da variável $S$ em consideração. O terceiro cumulante é uma medida de assimetria da distribuição de $S$ no sentido de que $\kappa_{3}$ é zero quando $S$ é distribuída simetricamente. Entretanto, $\kappa_{3}=0$ não é uma condição suficiente para $S$ ter distribuição simétrica. Para termos simetria, a distribuição deve ser unívocamente determinada pelos seus cumulantes e todos os cumulantes de ordem impar devem se anular. Os cumulantes de ordens maiores ou iguais a 3 podem ser interpretados como medidas de não-normalidade, pois eles se anulam quando $S$ é normal. Os cumulantes padronizados definidos por $\rho_{r}=\kappa_{r} / \kappa_{2}^{r / 2}$ para $r=1,2, \ldots$ são muito úteis na teoria assintótica (vide Seção 3.1).

\section{Expansão da FGM Através de uma Aproximação de Primeira Ordem.}

Consideramos uma estatística qualquer $S$ como na Seção 1. Seja $Z$ uma variável aleatória de referência usada para aproximar $S$. Os cumulantes de $Z$ são representados por $\kappa_{10}, \kappa_{20}$, etc. e as suas funções geratrizes de momentos e cumulantes são escritas como $M_{Z}\left(t ; \kappa_{0}\right)$ e $K_{Z}\left(t ; \kappa_{0}\right)$. Estas funções são uma primeira aproximação para as funções correspondentes $M_{S}(t ; \kappa)$ e $K_{S}(t ; \kappa)$ de $S$, cujos cumulantes são $\kappa_{1}, \kappa_{2}$, etc. Admitimos que os momentos e cumulantes das variáveis $S$ e $Z$ são finitos até uma ordem fixada. A expansão da função $K_{Z}\left(t ; \kappa_{0}\right)$ é análoga à equação (2). Na notação suprimiremos a dependência das funções geratrizes sobre os momentos e cumulantes.

A diferença entre $K_{S}(t)$ e $K_{Z}(t)$ pode ser expressa como

$$
I_{S} S(t)-K_{Z}(t)=\left(\kappa_{1}-\kappa_{10}\right) t+\cdots+\left(\kappa_{r}-\kappa_{r 0}\right) \frac{t^{r}}{r !}+\cdots
$$

e, portanto, a FGM de $S$ é dada por

$$
M_{S}(t)=M_{Z}(t) \exp \left\{\left(\kappa_{1}-\kappa_{10}\right) t+\cdots+\left(\kappa_{r}-\kappa_{r 0}\right) \frac{t^{r}}{r !}+\cdots\right\} .
$$


Metodologia para corrigir distribuiçōes de estatísticas

A expansão em série de Taylor da função exponencial na equação (3) implica

$$
M_{S}(t)=M_{Z}(t)\left\{1+\eta_{1} t+\cdots+\eta_{r} \frac{t^{r}}{r !}+\cdots\right\},
$$

com os $\eta_{i}^{\prime} s$ sendo obtidos como pseudo-momentos ordinários tratando as diferenças $\kappa_{i}-\kappa_{i 0}$ como pseudo-cumulantes. Enfatizamos que diferenças entre cumulantes correspondentes de $S$ e $Z$ não são, em geral, cumulantes de alguma variável aleatória. Por exemplo, $\kappa_{2}-\kappa_{20}$ não é necessariamente positivo. Logo, a terminologia adotada para as diferenças $\left(\kappa_{i}-\kappa_{i 0}\right)^{\prime} s$ como pseudo-cumulantes e para os $\eta_{i}^{\prime} s$ como pseudo-momentos é natural. Claro que os $\eta_{i}^{\prime} s$ são calculados dos $\left(\kappa_{i}-\kappa_{i 0}\right)^{\prime} s$ via as equações que expressam momentos ordinários como funções dos cumulantes (vide Seção 1). Os 6 primeiros $\eta_{i}^{\prime} s$ são:

$$
\begin{aligned}
\eta_{1} & =\kappa_{1}-\kappa_{10}, \eta_{2}=\kappa_{2}-\kappa_{20}+\left(\kappa_{1}-\kappa_{10}\right)^{2}, \\
\eta_{3} & =\kappa_{3}-\kappa_{30}+3\left(\kappa_{2}-\kappa_{20}\right)\left(\kappa_{1}-\kappa_{10}\right)+\left(\kappa_{1}-\kappa_{10}\right)^{3}, \\
\eta_{4} & =\kappa_{4}-\kappa_{40}+4\left(\kappa_{3}-\kappa_{30}\right)\left(\kappa_{1}-\kappa_{10}\right)+3\left(\kappa_{2}-\kappa_{20}\right)^{2} \\
& +6\left(\kappa_{2}-\kappa_{20}\right)\left(\kappa_{1}-\kappa_{10}\right)^{2}+\left(\kappa_{1}-\kappa_{10}\right)^{4}, \\
\eta_{5} & =\kappa_{5}-\kappa_{50}+5\left(\kappa_{4}-\kappa_{40}\right)\left(\kappa_{1}-\kappa_{10}\right)+10\left(\kappa_{3}-\kappa_{30}\right)\left(\kappa_{2}-\kappa_{20}\right) \\
& +10\left(\kappa_{3}-\kappa_{30}\right)\left(\kappa_{1}-\kappa_{10}\right)^{2}+15\left(\kappa_{2}-\kappa_{20}\right)^{2}\left(\kappa_{1}-\kappa_{10}\right) \\
& +10\left(\kappa_{2}-\kappa_{20}\right)\left(\kappa_{1}-\kappa_{10}\right)^{3}+\left(\kappa_{1}-\kappa_{10}\right)^{5}, \\
\eta_{6} & =\kappa_{6}-\kappa_{60}+6\left(\kappa_{5}-\kappa_{50}\right)\left(\kappa_{1}-\kappa_{10}\right)+15\left(\kappa_{4}-\kappa_{40}\right)\left(\kappa_{2}-\kappa_{20}\right) \\
& +15\left(\kappa_{4}-\kappa_{40}\right)\left(\kappa_{1}-\kappa_{10}\right)^{2}+10\left(\kappa_{3}-\kappa_{30}\right)^{2} \\
& +60\left(\kappa_{3}-\kappa_{30}\right)\left(\kappa_{2}-\kappa_{20}\right)\left(\kappa_{1}-\kappa_{10}\right)+20\left(\kappa_{3}-\kappa_{30}\right)\left(\kappa_{1}-\kappa_{10}\right)^{3} \\
& +15\left(\kappa_{2}-\kappa_{20}\right)^{3}+45\left(\kappa_{2}-\kappa_{20}\right)^{2}\left(\kappa_{1}-\kappa_{10}\right)^{2} \\
& +15\left(\kappa_{2}-\kappa_{20}\right)\left(\kappa_{1}-\kappa_{10}\right)^{4}+\left(\kappa_{1}-\kappa_{10}\right)^{6} .
\end{aligned}
$$


A expansão infinita (4) pode ser aproximada por uma soma finita

$$
M_{S}(t)=M_{Z}(t) \sum_{i=0}^{m} \eta_{i} \frac{t^{i}}{i !},
$$

sendo $\eta_{0}=1$ e com os $\eta_{i}^{\prime} s$ obtidos das equações em (5), onde $m$ é um inteiro positivo suficientemente grande, supondo que os $\eta_{i}^{\prime} s$ decrescem quando $i$ cresce. Em geral, a escolha de $m$ visa a produzir na equação (6) um erro de dada inagnitude. A interpretação da equação (6) é que a FGM de uma variável aleatória qualquer pode ser expandida em série de potências cujos coeficientes representam pseudo-momentos correspondentes às diferenças $\left(\kappa_{i}-\kappa_{i 0}\right)^{\prime} s$ tratadas como pseudo-cumulantes. Esta equação é bastante geral no sentido de que a FGM da variável de referência $Z$ pode ser escolhida arbitrariamente. Expressando as ordens das diferenças $\kappa_{i}-\kappa_{i 0}$ em termos do tamanho da amostra $n$, a escolha usual é tomar $M_{Z}(t)$ como $\lim _{n \rightarrow \infty} M_{S}(t)$, quando este limite existe e é conhecido, ou seja, $S$ convergindo em distribuição para $Z$ à medida que $n \rightarrow \infty$. Entretanto, $Z$ pode ser qualquer outra variável aleatória, escolhendoa, por exemplo, visando a minimizar o número de termos em (6) e a garantir que a ordem do erro desta soma seja menor do que um valor especificado (vide discussão no parágrafo anterior às equações (10) e (11)).

Para mostrar a generalidade da fórmula (6), obteremos a expansão da FGM da estatística escore, amplamentente usada em testes econométricos segundo a terminologia de testes multiplicadores de Lagrange, dada em Harris (1985; equação (3.1)), a partir desta fórmula. Salientamos que Harris calculou $M_{S_{R}}(t)$ por expansão direta de $E\left\{\exp \left(t S_{R}\right)\right\}$. Seja $M_{q}(t)=(1-2 t)^{-q / 2}$ a FGM de uma variável $\chi_{q}^{2}$. Harris (1985) demonstrou, até $O\left(n^{-1}\right)$, que

$$
\begin{aligned}
M_{S_{R}}(t)= & M_{q}(t)+\frac{1}{24}\left\{\left(A_{2}-A_{1}-A_{3}\right) M_{q}(t)+\left(A_{1}-2 A_{2}+3 A_{3}\right) \times\right. \\
& \left.M_{q+2}(t)+\left(A_{2}-3 A_{3}\right) M_{q+4}(t)+A_{3} M_{q+6}(t)\right\}
\end{aligned}
$$

Revista de Econometria 
onde os $A_{i}^{\prime} s$ são dados no artigo do Harris como funções de cumulantes de derivadas da log-verossimilhança.

A demonstração da equivalência entre as equações (6) e (7) é baseada no fato de que os coeficientes correspondentes a qualquer potência de $t$ nas duas expansões são iguais. Para usarmos este fato precisamos trabalhar com a expansão binomial $(1-2 t)^{m}=1-2 m t+$ $2 m(m-1) t^{2}-\frac{4}{3} m(m-1)(m-2) t^{3}+\cdots$ nas funções geratrizes de momentos $M_{g}(t), M_{q+2}(t), M_{q+4}(t)$ e $M_{q+6}(t)$. Usamos a notação $q^{(i)}=q(q+2) \cdots(q+2 i-2)$ para $i=1,2, \ldots$ De $(7)$ temos,

$$
\begin{aligned}
& M_{S_{R}}(t)=M_{q}(t)+\frac{1}{2} \\
& +\left(A_{1}-2 A_{2}+3 A_{3}\right)\left[1+(q+2) t+(q+2)^{(2)} \frac{t^{2}}{2 !}+(q+2)^{(3)} \frac{t^{3}}{3 !}+\cdots\right] \\
& +\left(A_{2}-3 A_{3}\right)\left[1+(q+4) t+(q+4)^{(2)} \frac{t^{2}}{2 !}+(q+4)^{(3)} \frac{t^{3}}{3 !}+\cdots\right] \\
& \left.+A_{3}\left[1+(q+6) t+(q+6)^{(2)} \frac{t^{2}}{2 !}+(q+6)^{(3)} \frac{t^{3}}{3 !}+\ldots\right]\right\} .
\end{aligned}
$$

Pode-se verificar, facilmente, que o termo independente de $t$ é nulo e que os coeficientes dos termos em $t, t^{2}$ e $t^{3}$ são, respectivamente, $A_{1} / 12, q A_{1} / 12+\left(A_{1}+A_{2}\right) / 6$ e $\left\{A_{1} q^{2} / 24+\left(3 A_{1}+2 A_{2}\right) q / 12+\left(A_{1}+\right.\right.$ $\left.\left.2 A_{2}+A_{3}\right) / 3\right\}$. O coeficiente $c_{r}$ de $t^{r}$ na expansão de Harris $M_{S_{R}}(t)=$ $M_{q}(t)+c_{1} t+c_{2} t^{2}+\cdots+c_{r} t^{r}+\cdots \dot{\mathrm{e}}$

$$
\begin{aligned}
c_{r}= & \left\{\left(A_{2}-A_{1}-A_{3}\right) q^{(r)}+\left(A_{1}-2 A_{2}+3 A_{3}\right)(q+2)^{(r)}\right. \\
& \left.+\left(A_{2}-3 A_{3}\right)(q+4)^{(r)}+A_{3}(q+6)^{(r)}\right\} /(24 r !) .
\end{aligned}
$$

Para obtermos a expansão da FGM da estatística escore via a equação (6), observamos que no caso de estatísticas cuja distribuição assintótica é qui-quadrado, temos $\kappa_{i}=\kappa_{i 0}+O\left(n^{-1}\right)$ e, portanto, das equações (5) concluímos que $\eta_{i}=\kappa_{i}-\kappa_{i_{0}}+O\left(n^{-2}\right)$ para $i=$ $1,2, \ldots$ Logo, até $O\left(n^{-1}\right)$, os $\eta_{i}^{\prime} s$ são simplesmente iguais aos termos de ordem $n^{-1}$ dos respectivos cumulantes $\kappa_{i}^{\prime} s$ de $S$. No caso da 
estatística escore, temos: $\eta_{1}=A_{1} / 12, \eta_{2}=\left(A_{1}+A_{2}\right) / 3$ e $\eta_{3}=$ $2\left(A_{1}+2 A_{2}+A_{3}\right)$. Da equação (6) vem:

$$
\begin{aligned}
M_{S_{R}}(t)= & \left\{1+q t+q^{(2)} \frac{t^{2}}{2 !}+q^{(3)} \frac{t^{3}}{3 !}+\cdots\right\} \times \\
& \left\{1+\eta_{1} t+\eta_{2} \frac{t^{2}}{2 !}+\eta_{3} \frac{t^{3}}{3 !}+\cdots\right\}
\end{aligned}
$$

ou, ainda,

$$
M_{S_{R}}(t)=M_{q}(t)+\eta_{1} t+\left(\eta_{1} q+\frac{\eta_{2}}{2 !}\right) t^{2}+\left(q^{(2)} \frac{\eta_{1}}{2 !}+q \frac{\eta_{2}}{2 !}+2 \frac{\eta_{3}}{3 !}\right) t^{3}+\cdots
$$

Substituindo os $\eta_{i}^{\prime} s$ acima, concluímos, que os coeficientes de $t, t^{2}$ e $t^{3}$ coincidem com aqueles correspondentes obtidos via (7). Com alguma álgebra, pode-se demonstrar também que o coeficiente $c_{r}^{\prime}$ da potência $t^{r}$ na última equação, dado por

$$
\left\{\frac{\eta_{r}}{r !}+\frac{q \eta_{r-1}}{(r-1) !}+\frac{q^{(2)} \eta_{r-2}}{2 !(r-2) !}+\cdots+\frac{q^{(r-1)} \eta_{1}}{(r-1) ! 1 !}\right\}
$$

coincide com o $c_{r}$ dado antes. Assim, mostramos como obter a expansão (7) da fórmula geral (6).

\section{Expansões do Tipo Edgeworth para a Densidade e Dis- tribuição de uma Estatística Qualquer.}

Nas expansões a seguir, consideramos que a estatística $S$ é contínu, cujas funções densidade e distribuição acumulada são deriváveis. Representamos, às vezes, as funções densidade e distribuição de $S$ por $f_{S}(x ; \kappa)$ e $F_{S}(x ; \kappa)$, respectivamente, para reforçar a dependência destas funções sobre os cumulantes $\kappa^{\prime} s$ de $S$. As expansões em série do tipo Edgeworth para a densidade de $S$, ou equivalentemente, para a sua distribuição acumulada, envolvem uma aproximação de primeira ordem inicial multiplicada por uma soma de 
termos representando correções, cujos coeficientes são combinações simples de diferenças entre os cumulantes de $S$ e da variável contínua $Z$ usada como aproximação de primeira ordem. O caso da expansão de Edgeworth se refere, usualmente, àquele caso em que $Z$ é normal. Quando isto ocorre, as correções são dadas por combinações lineares dos polinõmios de Hermite. Genericamente, usamos a terminologia expansōes do tipo Edgeworth quando a variável de referência não é normal.

No intuito de minimizar o número de termos nas expansões da densidade e distribuição de $S$, pode ser bem mais vantajoso usarmos aproximações de primeira ordem diferentes da normal, como por exemplo, a distribuição limite de $S$ em grandes amostras. As correções correspondentes não são, necessariamente, polinômios.

A suposição básica adicional aqui se refere à densidade $f_{\boldsymbol{Z}}\left(x ; \kappa_{0}\right)$ da variável de referência $Z$ que deve ter derivadas contínuas em todo suporte de $S$. Representamos as derivadas de $f_{Z}\left(x ; \kappa_{0}\right)$ por $D^{i} f_{Z}\left(x ; \kappa_{0}\right)$ para $i=1,2, \ldots$ A idéia da inversão da expansão da FGM de $S$ em (6) para obtermos a expansão correspondente de $f_{S}(x ; \kappa)$ é muito simples (vide Davis, 1976). Considere-se que $S=Z+T$, onde $Z$ tem densidade $f_{Z}\left(x ; \kappa_{0}\right)$ e $T$ é uma pseudovariável aleatória independente de $Z$ com "cumulantes" $\left(\kappa_{i}-\kappa_{i 0}\right)^{\prime} s$ e "momentos" $\eta_{i}^{\prime} s$. A terminologia de pseudo-variável aleatória está dada em Davis (1976). Esta variável não é uma variável aleatória genuína, pois os seus cumulantes não seguem as equações clássicas em termos dos momentos. Por exemplo, o segundo cumulante (que é a variância) da pseudo-variável aleatória pode ser negativo. Condicionalmente a $T=t, \quad S$ tem densidade $f_{Z}\left(x-t ; \kappa_{0}\right)$ e, portanto, a densidade marginal de $S$ é $f_{S}(x ; \kappa)=E_{T}\left\{f_{Z}\left(x-T ; \kappa_{0}\right)\right\}$. Expandindo em série de Taylor $E_{T}\left\{f_{Z}\left(x-T ; \kappa_{0}\right)\right\}$ em $T=0$ e tomando o valor esperado, obtém-se, até um certo número $m$ de termos,

$$
f_{S}(x ; \kappa)=f_{Z}\left(x ; \kappa_{0}\right)+\sum_{i=1}^{m} \frac{(-1)^{i}}{i !} \eta_{i} D^{i} f_{Z}\left(x ; \kappa_{0}\right)
$$


Assim, para calcularmos a expansão da densidade de $S$ precisamos apenas conhecer as derivadas da densidade de referência e os cumulantes de $S$ e $Z$, todos até uma ordem especificada. A fórmula (8) também poderia ser obtida, invertendo a equação (6) termo a termo. Esta equação se assemelha a uma expansão em Taylor, pois envolve quocientes de derivadas da função densidade de referência pelos fatoriais correspondentes, com a única particularidade que os coeficientes são iguais aos pseudo-momentos $\eta_{i}^{\prime} s$. A partir da densidade de uma variável de referência $Z$ e de seus cumulantes, pode-se construir, facilmente, a expansão da densidade de uma estatística qualquer $S$, desde que os cumulantes desta estatística sejam conhecidos. A equação (8) é conhecida como expansão do tipo Edgeworth para a densidade de $S$. No caso em que $f_{Z}\left(x ; \kappa_{0}\right)$ não é contínua, a expansão para $f_{S}(x ; \kappa)$ é muito complicada e apareceriam termos adicionais nesta equação.

A expansão do tipo Edgeworth para a distribuição de $S$ é obtida integrando (8). Temos,

$$
F_{S}(x ; \kappa)=F_{Z}\left(x ; \kappa_{0}\right)+\sum_{i=1}^{m} \frac{(-1)^{i}}{i !} \eta_{i} D^{i} F_{Z}\left(x ; \kappa_{0}\right) .
$$

Assim, basta substituirmos a densidade $f_{Z}\left(x ; \kappa_{0}\right)$ pela distribuição acumulada $F_{Z}\left(x ; \kappa_{0}\right)$ para obtermos de $(8)$ a equação $(9)$.

As equações (8) e (9) mostram que é possivel obtermos expansões para as funções densidade e distribuição de qualquer estatística contínua $S$, supondo que algumas condições de regularidade sejam satisfeitas, até uma certa ordem de aproximação, a partir do conhecimento dos cumulantes de $S$ e $Z$ e das derivadas da distribuição de referência $F_{Z}\left(x ; \kappa_{0}\right)$. As condições de regularidade são dadas em Skovgaard (1981a,b,1986).

O número $m$ de termos nas equações (6) e (8)-(9) é, usualmente, escolhido de modo que estas somas estejam corretas até uma ordem desejada. Caso as somas destas equações não fossem limitadas a $m$ 
termos, mas constituissem somas infinitas, estas poderiarn ser divergentes. Por exemplo, se todas as diferenças $\kappa_{i}-\kappa_{i 0}$ se igualam a um, das equações em (5) vemos que $\eta_{1}=1, \eta_{2}=2, \eta_{3}=5, \eta_{4}=15$, $\eta_{5}=52, \eta_{6}=203$, etc., números estes que são conhecidos como números de Bell. Estes números aumentam rapidamente e, apesar dos fatoriais nos denominadores, poderão implicar que as séries em (6) e (8)-(9) sejam não-convergentes. Por esta razão, justificamos o uso de expansões truncadas vinculando-as ao tamanho $n$ da amostra. A idéia de usarmos séries truncadas em (6) e (8)-(9) com $m$ termos é que, segundo condições de regularidade adequadas, podemos encontrar o erro destas equações. Por exemplo, em um grande número de aplicações, $S$ é uma soma padronizada de variáveis aleatórias independentes e $Z$ é a normal reduzida de mesma média e variância que $S$. Então, $\kappa_{1}-\kappa_{10}=\kappa_{2}-\kappa_{20}=0$ e $\kappa_{i}-\kappa_{i 0}=O\left(n^{-(i-2) / 2}\right)$ para $i \geq 3$. Pode-se verificar que o erro nas equações (6) e (8)-(9) com $m$ termos é, tipicamente, de ordem $O\left(n^{-(m-3) / 2}\right)$. Para o erro ser de ordem $O\left(n^{-3 / 2}\right)$ teremos que computar somas com 6 termos. As expansões (8)-(9) não são válidas para variáveis aleatórias discretas pois, nestes casos, $F_{S}(x ; \kappa)$ tem descontinuidades com probabilidades associadas de ordem $O\left(n^{-1 / 2}\right)$. Qualquer aproximação contínua para $F_{S}(x ; \kappa)$ tem, portanto, erro desta ordem, não importanto o número $m$ de termos. Podemos expressar (8) e (9) como correções multiplicativas para $f_{Z}\left(x ; \kappa_{0}\right)$ e $F_{Z}\left(x ; \kappa_{0}\right)$, respectivamente. Temos,

$$
f_{S}(x ; \kappa)=f_{Z}\left(x ; \kappa_{0}\right)\left\{1+\sum_{i=1}^{m} \frac{(-1)^{i}}{i !} \eta_{i} h_{i}(x)\right\}
$$

e

$$
F_{S}(x ; \kappa)=F_{Z}\left(x ; \kappa_{0}\right)\left\{1+\sum_{i=1}^{m} \frac{(-1)^{i}}{i !} \eta_{i} H_{i}(x)\right\}
$$

onde $h_{i}(x)=(-1)^{i} D^{i} f_{Z}\left(x ; \kappa_{0}\right) / f_{Z}\left(x ; \kappa_{0}\right)$ e $H_{i}(x)=(-1)^{i} D^{i}$ $F_{Z}\left(x ; \kappa_{0}\right) / F_{Z}\left(x ; \kappa_{0}\right)$. 
Em muitas situações, as funções $h_{i}(x)$ e $H_{i}(x)$ têm propriedades matemáticas interessantes, como será visto a seguir.

\subsection{Série de Gram Charlier.}

Um caso importante das equações (10)-(11) é obtido supondo que a variável aleatória de referência é a normal padrão, i.e.,

$$
f_{Z}\left(x ; \kappa_{0}\right)=\phi(x)=\frac{1}{\sqrt{2 \pi}} \exp \left(-x^{2} / 2\right) .
$$

Neste caso, os $h_{i}(x)^{\prime} s$ reduzem-se aos polinômios de Hermite: $h_{1}(x)=x, h_{2}(x)=x^{2}-1, h_{3}(x)=x^{3}-3 x, h_{4}(x)=x^{4}-6 x^{3}+3$, $h_{5}(x)=x^{5}-10 x^{3}+15 x, h_{6}(x)=x^{6}-15 x^{4}+45 x^{2}-15$, etc. Fazendo concordar os dois primeiros momentos de $S$ com aqueles correspondentes de $Z$, ou seja, $\kappa_{1}=0$ e $\kappa_{2}=1$, sabendo que $\kappa_{i 0}=0$ para $i \geq 3$, encontramos de (10), usando (5), a série de Gram Charlier

$$
\begin{aligned}
f_{S}(x ; \kappa)= & \phi(x)\left\{1+\frac{\kappa_{3}}{6} h_{3}(x)+\frac{\kappa_{4}}{24} h_{4}(x)+\frac{\kappa_{5}}{120} h_{5}(x)\right. \\
& \left.+\frac{\left(\kappa_{6}+10 \kappa_{3}^{2}\right)}{720} h_{6}(x)+\cdots\right\} .
\end{aligned}
$$

Uma aplicação importante da fórmula (12) é relativa à expansão da função densidade de uma soma padronizada de variáveis aleatórias independentes e identicamente distribuídas (IID). Sejam $X_{1}, \ldots, X_{n}$ variáveis contínuas IID cujos cumulantes comuns e cumulantes padronizados são $\delta_{r}$ e $\rho_{r}=\delta_{r} / \delta_{2}^{r / 2}$, respectivamente. Definimos a estatística $S=\left(\sum_{i=1}^{n} X_{i}-n \delta_{1}\right) /\left(n \delta_{2}\right)^{1 / 2}$ que satisfaz às condições acima. A fórmula anterior determina as ordens dos cumulantes $\kappa_{r}^{\prime} s$ de $S$, i.e., $\kappa_{r}=O\left(n^{-(r-2) / 2}\right)$ para $r \geq 3$. Revertendo os $\kappa^{\prime} s$ na equação (12) para os $\rho^{\prime} s$, obtemos até $O\left(n^{-1}\right)$ :

$$
f_{S}(x ; \kappa)=\phi(x)\left\{1+\frac{\rho_{3} h_{3}(x)}{6 \sqrt{n}}+\frac{1}{n}\left[\frac{\rho_{4} h_{4}(x)}{24}+\frac{\rho_{3}^{2} h_{6}(x)}{72}\right]\right\},
$$

Revista de Econometria 
onde o erro desta equação é $O\left(n^{-3 / 2}\right)$.

Tecemos alguns comentários sobre a fórmula (13). O termo principal desta equação é a densidade normal pelo teorema do limite central. O termo de ordem $n^{-1 / 2}$ é o ajustamento para a assimetria através de $\rho_{3}$ e o termo de ordem $n^{-1}$ é um ajustamento simultãneo para assimetria e curtose. Se a densidade dos $X_{i}^{\prime} s$ é simétrica, $\rho_{3}=0$ e a aproximação normal está correta até ordem $n^{-1}$ ao invés da ordem usual $n^{-1 / 2}$ quando $\rho_{3} \neq 0$. A ordem do erro da aproximação (13), embora seja usualmente $O\left(n^{-3 / 2}\right)$, pode depender do valor de $x$. Para $x=0$, a ordem do erro é reduzida para $O\left(n^{-2}\right)$. Em especial, a aproximação (13) é ruim (e pode mesmo ser negativa) nas extremidades da densidade, isto é, quando $|x|$ cresce, porque os polinômios de Hermite não são limitados.

\subsection{Distribuição Limite $\chi_{q}^{2}$.}

Outro caso especial de interesse da equação (10) (ou (11)) referese às estatísticas que têm distribuição assintótica qui-quadrado com $q$ graus de liberdade, ou seja,

$$
f_{q}(x)=\left\{2^{q} \Gamma(q / 2)\right\}^{-1} x^{(q-2) / 2} \exp (-x / 2) .
$$

Tomamos, então, esta distribuição como de referência. As densidades qui-quadrados satisfazem às equações

$$
\frac{d^{m}}{d x^{m}} f_{q}(x)=\frac{1}{2^{m}} \sum_{j=0}^{m}(-1)^{m+j} f_{q-2 j}(x)
$$

e

$$
f_{q+2 l}(x)=\frac{\Gamma(q / 2) x^{l} f_{q}(x)}{2^{l} \Gamma(q / 2+l)} .
$$

Destas equações podemos demonstrar, com algum algebrismo, que $h_{i}(x)=2^{-i} \sum_{j=0}^{i} \frac{(-1)^{j} v_{j}}{x^{j}}$, onde $v_{j}=\Pi_{r=1}^{j}(q-2 r)$ se $j \geq 1$ e $v_{0}=1$. 
De forma análoga, $h_{i}(x)$ é dado por

$$
\begin{aligned}
h_{i}(x)=2^{-i} & \left\{1-\frac{(q-2)}{x}+\frac{(q-2)(q-4)}{x^{2}}+\cdots\right. \\
+ & \left.\frac{(-1)^{i}(q-2) \cdots(q-2 i)}{x^{i}}\right\} .
\end{aligned}
$$

A expansão da densidade de qualquer estatística $S$ até $O\left(n^{-1}\right)$, tendo distribuição assintótica qui-quadrado, é obtida de (10) com os $h_{i}(x)^{\prime} s$ dados por (14) e onde os $\eta_{i}^{\prime} s$ são os termos de ordem $n^{-1}$ dos cumulantes $\kappa_{i}^{\prime} s$ de $S$, ou seja, $\eta_{i}=\kappa_{i}-2^{i-1}(i-1) ! q, \quad i \geq 1$.

\section{Generalização das Correções.}

As correções desenvolvidas neste artigo são úteis, em particular, quando a aproximação da estatística em questão por uma dada distribuição de referência não é satisfatória. Isto ocorre, em geral, em amostras pequenas. No entanto, em muitos problemas práticos, o tamanho da amostra pode ser muito grande e, ainda assim, a aproximação envolvida não é razoável. Exemplos econométricos onde este fato ocorre referem-se, por exemplo, a testes de especificação. Chesher e Spady (1991), Davidson e MacKinnon (1992), Orme (1990) e Taylor (1987), entre outros, mostram que os tamanhos dos testes de especificação podem ser muito distorcidos em amostras bastante grandes. Cribari-Neto (1994, Capítulo 4), por sua vez, mostra a utilidade prática do uso de correções para testes de especificação. Não daremos aqui exemplos de cálculo da estatística corrigida. Entretanto, o leitor poderá consultar Cordeiro $(1993,1995)$ para exemplos de cálculo completo das correções de Bartlett em modelos normais heterocedásticos e modelos lineares generalizados, respectivamente. Estes exemplos comprovam que a utilização das correções de Bartlett tornam os testes modificados realmente mais precisos relativamente a aproximações assintóticas aos níveis de tamanhos de amostra que usualmente aparecem em Econometria. Em relação aos testes LM, 
exemplos de aprimoramento são dados em Cordeiro, Ferrari e Paula (1993). Enfatizamos que a área de pesquisa em correções de Bartlett ainda não é muito explorada pelos econometristas, apesar de merecer grande atenção dos estatísticos desde o início da década de 80 .

Nesta seção, apresentamos uma fórmula geral para corrigir qualquer estatística-teste contínua $S=S\left(X_{1}, \ldots, X_{n}\right)$, onde os $X_{i}^{\prime} s$ são variáveis aleatórias contínuas, não necessariamente independentes e identicamente distribuídas, tendo aproximação de $1 \underline{a}$ ordem especificada. Considere-se que os pseudo-momentos ordinários $\eta_{i}^{\prime} s$ estão relacionados com o tamanho da amostra $n$ de modo que suas ordens não-cresçam quando $i$ cresce. Este caso corresponde a um grande número de estatísticas-teste na prática. A partir da equação (9), podemos escrever a distribuição da estatística $S$ como

$$
F_{S}(x)=F_{Z}(x)+A_{1}(x)+A_{2}(x)+O\left(n^{-3 / 2}\right),
$$

onde $A_{1}(x)$ e $A_{2}(x)$ são termos $O\left(n^{-1 / 2}\right)$ e $O\left(n^{-1}\right)$, respectivamente, que dependem de certas diferenças entre cumulantes $\left(\kappa_{i}-\kappa_{i 0}\right)^{\prime}$ s. Suponhamos que $F_{Z}(x)$ tem derivadas contínuas para todo $x$ pertencente ao suporte de $S$.

O nosso objetivo é definir uma estatística-teste corrigida

$$
S^{*}=S-b_{1}(S)-b_{2}(S),
$$

onde $b_{i}(S)=O_{p}\left(n^{-i / 2}\right)$, de modo que tenhamos $F_{S^{*}}(x)=F_{Z}(x)$ até ordem $n^{-1}$. O teorema 1 de Cox e Reid (1987) permite determinar $F_{S^{*}}(x)$ até $O\left(n^{-1}\right)$ e, então, encontrar os termos $b_{1}(S)$ e $b_{2}(S)$ em (16). Deste teorema, temos até ordem $n^{-1}$

$$
\begin{aligned}
F_{S^{*}}(x)= & F_{S}(x)-E\left\{-b_{1}(S) \mid S=x\right\} f_{S}(x)-E\left\{-b_{2}(S) \mid S=x\right\} \\
& \times f_{S}(x)+\frac{1}{2} \frac{d}{d x}\left[E\left\{b_{1}(S)^{2} \mid S=x\right\} f_{S}(x)\right] .
\end{aligned}
$$

Logo, desta equação e de (15), concluímos que $F_{S^{*}}(x)$ iguala $F_{Z}(x)$ se, e somente se,

$$
A_{1}(x)+A_{2}(x)+b_{1}(x) f_{S}(x)+b_{2}(x) f_{S}(x)+\frac{1}{2} \frac{d}{d x}
$$


Da equação anterior, temos que

$$
A_{1}(x)+b_{1}(x) f_{Z}(x)=0
$$

e

$$
\begin{aligned}
A_{2}(x) & +b_{1}(x) A_{1}^{\prime}(x)+b_{2}(x) f_{Z}(x)+b_{1}(x) b_{1}^{\prime}(x) f_{Z}(x) \\
+ & \frac{1}{2} b_{1}(x)^{2} f_{Z}^{\prime}(x)=0
\end{aligned}
$$

onde as linhas são derivadas em relação a $x$.

O sistema de equações acima permite determinar as funções $b_{1}(\cdot)$ e $b_{2}(\cdot)$. Suponhamos que $f_{Z}(x)>0$ para todo $x$ no suporte de $S$. Da 1 a equação, temos $b_{1}(x)=-A_{1}(x) / f_{Z}(x)$. Substituindo $b_{1}(x)$ na $2^{\text {a }}$ equação, encontramos $b_{2}(x)=-A_{2}(x) / f_{Z}(x)+$ $A_{1}(x)^{2} f_{Z}^{\prime}(x) /\left\{2 f_{Z}(x)^{3}\right\}$. Da equação (16), obtemos a estatística corrigida,

$$
S^{*}=S\left\{1+\frac{A_{1}(S)}{f_{Z}(S) S}+\frac{1}{S}\left[\frac{A_{2}(S)}{f_{Z}(S)}-\frac{A_{1}(S)^{2} f_{Z}^{\prime}(S)}{2 f_{Z}(S)^{3}}\right]\right\} .
$$

Concluímos, então, que para corrigir qualquer estatística-teste até ordem $n^{-1}$, precisamos apenas conhecer certas diferenças de cumulantes $\kappa_{i}-\kappa_{i 0}$ até esta ordem, determinando da equação (11) $A_{1}(x)$ e $A_{2}(x)$, bem como, as derivadas $D^{i} F_{Z}(x)$. O termo entre chaves da equação (17) pode ser escrito como $1+b\left(S, \eta_{i}, D^{i} F_{Z}\right)$ e a estatística corrigida expressa por $S^{*}=S\left\{1+b\left(S, \eta_{i}, D^{i} F_{Z}\right)\right\}$. O fator multiplicativo $1+b\left(S, \eta_{i}, D^{i} F_{Z}\right)$ é um tipo de correşão de Bartlett generalizada aplicável a qualquer estatística-teste $S$ e densidade de referência $f_{Z}(x)$. Como citamos anteriormente, $Z$ pode ser qualquer variável escolhida como primeira aproximação de $S$, não sendo necessariamente igual à variável limite de $S$ quando $n \rightarrow \infty$. As restrições sobre $Z$ para usarmos (17) são aquelas mesmas restrições necessárias às equações (8) e (9).

Ao invés de modificarmos a estatística $S$, um método alternativo seria modificar os pontos críticos da distribuição de referência 
de modo que poderemos fazer uma melhor inferência baseado-se na própria estatística não-corrigida $S$. Da equação (2) de Cox e Reid (1987) e do fato de que $S^{*}$ em (17) tem função de distribuição igual a $F_{Z}(x)$ até ordem $n^{-1}$, podemos demonstrar que o ponto crítico modificado $x^{*}$, tal que $F_{S}\left(x^{*}\right)=F_{Z}(x)$, é dado por

$$
\begin{aligned}
x^{*} & =x\left[1-\frac{A_{1}(x)}{x f_{Z}(x)}-\frac{1}{x}\left\{-\frac{A_{1}(x) A_{1}^{\prime}(x)}{f_{Z}(x)^{2}}+\frac{A_{2}(x)}{f_{Z}(x)}\right.\right. \\
& \left.\left.+\frac{A_{1}(x)^{2} f_{Z}^{\prime}(x)}{2 f_{Z}(x)^{3}}\right\}\right] .
\end{aligned}
$$

Portanto, um inferência aperfeiçoada pode ser obtida de duas formas distintas, que são equivalentes até $O\left(n^{-1}\right)$. Na primeira, nós construímos a estatística corrigida $S^{*}$ que é melhor aproximada por $F_{Z}(x)$. Na segunda, nós obtemos uma nova distribuição de referência definida pelo ponto crítico modificado $x^{*}$ (vide equação anterior), que é mais próxima da distribuição verdadeira de $S$ do que a distribuição de $Z$. Claro que a dupla igualdade $F_{S^{*}}(x)=F_{S}\left(x^{*}\right)=F_{Z}(x)$ se verifica até $O\left(n^{-1}\right)$. As formas das correções multiplicativas para modificar os pontos críticos de $S$ e a própria estatística $S$ são, em geral, diferentes, a menos que o termo $A_{1}(x)$ de ordem $n^{-1 / 2}$ seja nulo.

\section{Casos Especiais.}

Deduzimos, agora, três resultados conhecidos como simples casos especiais da fórmula (17) para mostrar a generalidade e a importância desta fórmula. Iniciamos com o caso de grande interesse em que a aproximação de primeira ordem $Z$ é a normal reduzida. Neste caso, como vimos na Seção 3.2, a expansão da densidade da soma padronizada

$$
S=\left(\sum_{i=1}^{n} X_{i}-n \delta_{1}\right) /\left(\sqrt{n} \delta_{2}^{1 / 2}\right),
$$


onde os $X_{i}^{\prime} s$ são variáveis aleatórias contínuas IID com cumulantes padronizados $\rho_{r}=\delta_{r} / \delta_{2}^{r / 2}(r \geq 2)$, é dada, até ordem $n^{-1}$, pela equação (13). Observe-se que os cumulantes de $S$ são $\kappa_{r} S=$ $\rho_{r} n^{1-r / 2}, r \geq 2$. Verificamos isto usando a propriedade de que o cumulante de uma soma de variáveis aleatórias independentes iguala à soma dos cumulantes dessas variáveis.

Integrando a equação (13) e observando que

$$
\begin{aligned}
\int_{-\infty}^{v} \phi(x) h_{i}(x) d x & =\int_{-\infty}^{v}(-1)^{i} D^{i} \phi(x) d x=(-1)^{i} D^{i} \Phi(v) \\
& =-\phi(v) h_{i-1}(v),
\end{aligned}
$$

onde $\phi(\cdot)$ e $\Phi(\cdot)$ são as funções densidade e distribuição acumulada de $Z$, respectivamente, temos

$$
F_{S}(x, \kappa)=\Phi(x)-\phi(x)\left\{\frac{\rho_{3} h_{2}(x)}{6 \sqrt{n}}+\frac{1}{n}\left[\frac{\rho_{4} h_{3}(x)}{24}+\frac{\rho_{3}^{2} h_{5}(x)}{72}\right]\right\}
$$

A expansão da distribuição acumulada de qualquer soma padronizada $S$ até $O\left(n^{-1}\right)$ é, portanto, calculada de (18) com o conhecimento apenas dos terceiro e quarto cumulantes padronizados $\rho_{3}$ e $\rho_{4}$ das variáveis IID.

Combinando as equações (15) e (18), temos

$$
\begin{aligned}
& A_{1}(x)=-\rho_{3} \phi(x) h_{2}(x) / 6 \sqrt{n}, \\
& A_{2}(x)=-\phi(x)\left[\rho_{4} h_{3}(x) / 24+\rho_{3}^{2} h_{5}(x) / 72\right] / n .
\end{aligned}
$$

Substituindo estas funções na equação (17), obtém-se a estatística corrigida

$$
S^{*}=S-\frac{\rho_{3}}{6 n^{1 / 2}}\left(S^{2}-1\right)-\frac{\rho_{4}}{24 n}\left(S^{3}-3 S\right)+\frac{\rho_{3}^{2}}{36 n}\left(4 S^{3}-7 S\right) .
$$

Revista de Econometria 15(2) Novembro/Março 1996 
A fórmula (19) é justamente a clássica transformação polinomial de Cornish-Fisher que normaliza a distribuição de $S^{*}$ até ordem $O p\left(n^{-1}\right)$, isto é, $S^{*} \sim N(0,1)+O p\left(n^{-3 / 2}\right)$.

Como segundo caso especial, consideramos as estatísticas que têm distribuição assintótica igual a qui-quadrado. Neste caso, a expansão da distribuição acumulada de $S$ foi obtida por Chandra (1985) como $F_{S}(x)=F_{q}(x)+\sum_{l=0}^{k} a_{l} F(x)$, onde $F_{q}(x)$ é a distribuição acumulada da $\chi_{q}^{2}$ de referência e os $a_{l}^{\prime} s$ são funções de cumulantes de derivadas da log-verossimilhança. Usando (15), concluímos que

$$
A_{1}(x)=0 \quad \text { e } \quad A_{2}(x)=\sum_{l=0}^{k} a_{l} F_{q+2 l}(x) .
$$

Através da fórmula de recorrência entre acumuladas de quiquadrados

$$
F_{r+2}(x)=F_{r}(x)-\frac{2 x}{r} \frac{d F_{r}(x)}{d x}
$$

e da equação (17), chegamos à fórmula $S^{*}=S\left(1-\sum_{i=1}^{k} c_{i} S^{i-1}\right)$, onde

$$
c_{i}=2 \mu_{i}^{\prime-1} \sum_{l=i}^{k} a_{l}
$$

e $\mu_{i}^{\prime}=E\left\{\left(\chi_{q}^{2}\right)^{i}\right\}$ que foi deduzida por Cordeiro e Ferrari (1991; vide fórmula (16)). O caso $k=1$ corresponde à clássica correção de Bartlett.

Tratamos, agora, do caso das estatísticas $V$ que convergem em distribuição para uma combinação linear finita ou infinita de quiquadrados independentes, todas com um grau de liberdade, ou seja, $S \stackrel{\mathcal{D}}{\longrightarrow} \sum_{i=1}^{k} a_{i} \chi_{1 i}^{2}$, onde os $a_{i}^{\prime} s$ são números positivos. Assim, o $3^{\mathrm{O}}$ caso é uma extensão do $2^{\underline{Q}}$ caso, o qual corresponde a $a_{1}=\cdots=$ 
$a_{k}=1$. As estatísticas $V$ são, em geral, definidas a partir de $n$ variáveis $X_{1}, \ldots, X_{n}$ com distribuição comum $F_{X}$, por

$$
V=n^{-2} \sum_{i=1}^{n} \sum_{j=1}^{n} h\left(X_{i}, X_{j}\right)
$$

onde a função $h(x, y)$ é simétrica.

Sob condições apropriadas, $S=n V \stackrel{\mathcal{D}}{\longrightarrow} \sum_{i=1}^{k} a_{i} \chi_{1 i}^{2}$, onde $k$ pode ser $\infty$. Entre os exemplos das estatísticas $V$ constam as estatísticas de Cramér e Von-Mises (Durbin, 1973), o teste de um círculo de Watson (1961) e os testes consistentes baseados na transformação empírica de Laplace (Baringhauss e Henze, 1992). Não existe forma fechada para a distribuição $F_{V}$ de $(20)$ nem para a sua densidade $f_{V}$, mesmo quando $k$ é finito e apenas poucos $a_{i}^{\prime} s$ são diferentes de um. Neste sentido, Cordeiro e Pérez-Abreu (1995) ao invés de trabalharem com a expansão da distribuição de $V$, consideraram a expansão da função característica $C_{S}(t)=E\{\exp (i t S)\}$ de $S=n V$ supondo que esta existe para $t$ real em algum intervalo não-nulo contendo a origem. A função característica de $Z=\sum_{i=1}^{k} a_{i} \chi_{1 i}^{2}$ é $C_{Z}(t)=\Pi_{i=1}^{k}\left(1-2 i t a_{i}\right)^{-1 / 2}$.

Seja $P_{2}(t)$ o termo de ordem $n^{-1}$ na expansão de $C_{S}(t)$, ou seja, $C_{S}(t)=C_{Z}(t)\left\{1+P_{2}(t)\right\}+O\left(n^{-3 / 2}\right)$. Cordeiro e PérezAbreu demonstraram que $P_{2}(t)$ é a transformada de Fourier de medidas sinalizadas que representam combinações lineares finitas de convoluções de distribuições qui-quadrados. A fórmula de $P_{2}(t)$ é bastante complicada, sendo deixada à margem deste artigo. Este resultado generaliza o artigo de Chandra (1985) que corresponde ao caso em que todos os $a_{i}^{\prime} s$ são unitários. Neste caso, qualquer estatística - teste cuja distribuição nula assintótica é $\chi_{q}^{2}$ tem expansão dada por uma combinação linear finita de qui-quadrados com $q, q+2, \cdots, q+2 k$ graus de liberdade. Cordeiro e PérezAbreu (1995) mostraram, ainda, como obter a estatística corrigida $S^{*}=S\{1-b(S)\}$ que tem a mesma distribuição de $Z$ até $O\left(n^{-1}\right)$. 
A correção tipo-Bartlett $1-b(S)$ para $S=n V$ é obtida a partir de

$$
b(x)=\left(2 \pi x f_{Z}(x)\right)^{-1} \int_{0}^{x} \int_{0}^{\infty} e^{-i t y} C_{Z}(t) P_{2}(t) d t d y .
$$

Podemos comprovar, com alguma álgebra, que a equação (21) pode ser deduzida diretamente de (17). Para fazer isso, basta usarmos o teorema da inversão e transformarmos a expansão da distribuição de $S$ em termos da expansão de sua função característica.

\section{Aplicações Diversas.}

Nesta seção, apresentamos duas aplicações da equação (17) para corrigir as distribuições da função escore padronizada e da estimativa de máxima verossimilhança, ambas no contexto uniparamétrico. A grande importância destas fórmulas é construir, através da função escore padronizada e da estimativa de máxima verossimilhança, intervalos de confiança aperfeiçoados para o parâmetro de interesse. As fórmulas obtidas podem ser adaptadas a inúmeras situações uniparamétricas de interesse em Econometria e Estatística. Por exemplo, nos modelos não-lineares de regressão, o teste de hipótese sobre um parâmetro especificado pode ser construído condicionalmente, supondo que os demais parâmetros de incômodo estão fixados, usando-se a função escore padronizada correspondente a este parâmetro ou a estimativa de máxima verossimilhança restrita do parâmetro de interesse. Outras aplicações em Econometria seriam o teste da hipótese do erro estocástico seguir um processo autoregressivo de primeira ordem e o cálculo de intervalos de confiança aperfeiçoados para as autocorrelações parciais de um modelo ARIMA.

Seja um problema regular uniparamétrico cuja log-verossimilhança total para $\theta$ é $l(\theta)$. Fazemos, agora, uso da notação clássica de cumulantes de derivadas da log-verossimilhança $l(\theta)$ :

$$
\begin{array}{ll}
\kappa_{\theta \theta}=E\left(d^{2} l(\theta) / d \theta^{2}\right), & \kappa_{\theta, \theta}=E\left\{(d l(\theta) / d \theta)^{2}\right\}, \\
\kappa_{\theta \theta \theta}=E\left(d^{3} l(\theta) / d \theta^{3}\right), & \kappa_{\theta, \theta \theta}=E\left\{d l(\theta) / d \theta d^{2} l(\theta) / d \theta^{2}\right\}, \ldots .
\end{array}
$$


Sejam, ainda,

$$
\kappa_{\theta \theta}^{(\theta)}=d \kappa_{\theta \theta} / d \theta, \quad \kappa_{\theta \theta}^{(\theta \theta)}=d^{2} \kappa_{\theta \theta} / d \theta^{2}, \cdots,
$$

as derivadas destes cumulantes. A função escore total é $U(\theta)=$ $d l(\theta) / d \theta$, cuja esperança é nula e cuja variãncia se iguala à informação total de Fisher $\kappa_{\theta, \theta}$. A função escore padronizada $S=$ $U(\theta) / \kappa_{\theta, \theta}^{1 / 2}$ é, frequentemente, usada para construir intervalos de confiança para $\theta$ com erro de ordem $n^{-1 / 2}$. Nosso objetivo é construir intervalos para $\theta$ com erro $O\left(n^{-3 / 2}\right)$. Como antes, sejam $\kappa_{r S}$ e $\kappa_{r U}$ os cumulantes de $S$ e $U$, respectivamente. Como $S$ é uma soma de $n$ contribuições individuais dividida pela raiz quadrada da informação total, então $S$ satisfaz a soma padronizada definida no início da Seção 5 , com seus cumulantes dados por $\kappa_{r S}=\kappa_{r U} \kappa_{\theta, \theta}^{-r / 2}$. Em particular, $\kappa_{1 S}=0$ e $\kappa_{2 S}=1$. Assim, a equação (19) permanece válida para $S=U(\theta) \kappa_{\theta, \theta}^{-1 / 2}$, ou simplesmente, $S=U \kappa_{\theta, \theta}^{-1 / 2}$, com $\rho_{3} / \sqrt{n}$ e $\rho_{4} / n$ sendo substituídos por $\kappa_{3 S}=\kappa_{3 U} \kappa_{\theta, \theta}^{-3 / 2}$ e $\kappa_{4} S=\kappa_{4} U \kappa_{\theta, \theta}^{-2}$, respectivamente. Os 3 ㅇ e 4 을 cumulantes de $U$ são calculados através das clássicas identidades de Bartlett:

$$
\begin{aligned}
& \kappa_{3 U}=2 \kappa_{\theta \theta \theta}+3 \kappa_{\theta, \theta}^{(\theta)}, \\
& \kappa_{4 U}=6 \kappa_{\theta, \theta}^{(\theta \theta)}+8 \kappa_{\theta \theta \theta}^{(\theta)}-3 \kappa_{\theta \theta \theta \theta}+3 \operatorname{Var}\left(\frac{d^{2} l(\theta)}{d \theta^{2}}\right) .
\end{aligned}
$$

Logo, da equação (19), chegamos à fórmula da função escore aperfeiçoada

$$
\begin{aligned}
S^{*}= & \kappa_{\theta, \theta}^{-1 / 2}\left\{U-\frac{\kappa_{3 U}}{6 \kappa_{\theta, \theta}^{2}}\left(U^{2}-\kappa_{\theta, \theta}\right)-\frac{\kappa_{4} U}{24 \kappa_{\theta, \theta}^{3}}\left(U^{3}-3 \kappa_{\theta, \theta} U\right)\right. \\
& \left.+\frac{\kappa_{3 U}^{2}}{36 \kappa_{\theta, \theta}^{4}-}\left(4 U^{3}-7 \kappa_{\theta, \theta} U\right)\right\} .
\end{aligned}
$$

Revista de Econometria 15(2) Novembro/Março 1996 
$\mathrm{O}$ erro da fórmula (22) é $O_{p}\left(n^{-3 / 2}\right)$. Esta equação é muito conhecida na literatura (vide, por exemplo, Kendall e Stuart, 1977; pág. 124). Calculando $U, \kappa_{\theta, \theta}, \kappa_{3} U$ e $\kappa_{4} U$ para qualquer modelo uniparamétrico, podemos obter intervalos de confiança corretos até $O\left(n^{-3 / 2}\right)$ para o parâmetro $\theta$ deste modelo, por simples inversâo de

$$
P\left(\left|S^{*}\right| \leq k_{\alpha}\right)=1-\alpha \quad \text { com } \quad k_{\alpha}=\Phi^{-1}(1-\alpha / 2) .
$$

Passamos, agora, a corrigir a distribuição da estimativa de máxima verossimilhança $(E M V) \hat{\theta}$ do parâmetro único $\theta$ num problema regular. Trabalhamos com a estatística padronizada $S=$ $(\hat{\theta}-\theta) \kappa_{\theta, \theta}^{1 / 2}$. Em primeiro lugar, deduzimos de (9) a expansão da distribuiçâo acumulada de $S$ até $O\left(n^{-1}\right)$ tomando $Z \sim N(0,1)$ como

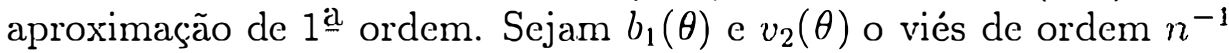
e a variância de ordem $n^{-2}$, respectivamente, da $E M I V \hat{\theta}$. Temos, $E(\hat{\theta})=\theta+b_{1}(\theta)+O\left(n^{-2}\right)$ c $\operatorname{Var}(\hat{\theta})=\kappa_{\theta, \theta}^{-1}+v_{2}(\theta)+O\left(n^{-3}\right)$. Sejam, ainda, $\rho_{3 \dot{\theta}}$ e $\rho_{4 \dot{\theta}}$ os $3 \stackrel{\bullet}{\text { c }} 4 \boldsymbol{\bullet}$ cumulantes padronizados de $\hat{\theta}$ de ordens $n^{-1 / 2}$ e $n^{-1}$, respectivamente. Existem fórmulas na literatura para encontrarmos, em generalidade, $b_{1}(\theta), v_{2}(\theta), \rho_{3 \dot{\theta}}$ e $\rho_{4 \dot{\theta}}$. Estas fórmulas serão vistas mais adiante.

Como $\kappa_{1 Z}=0, \kappa_{2 Z}=1, \kappa_{r} Z=0, r \geq 3$, encontramos das equações (5) com alguma álgebra,

$$
\begin{aligned}
& \eta_{1}=\kappa_{\theta, \theta}^{1 / 2} b_{1}(\theta)+O\left(n^{-3 / 2}\right), \\
& \eta_{2}=\kappa_{\theta, \theta}\left\{v_{2}(\theta)+b_{1}(\theta)^{2}\right\}+O\left(n^{-2}\right), \\
& \eta_{3}=\rho_{3 \dot{\theta}}+O\left(n^{-3 / 2}\right), \\
& \eta_{4}=\rho_{4 \dot{\theta}}+4 \rho_{3 \dot{\theta}} \kappa_{\theta, \theta}^{1 / 2} b_{1}(\theta)+O\left(n^{-2}\right) .
\end{aligned}
$$

Assim, $\eta_{1}, \eta_{2}, \eta_{3}$ e $\eta_{4}$ são quantidades calculadas até as ordens $n^{-1 / 2}, n^{-1}, n^{-1 / 2}$ e $n^{-1}$, respectivamente. Pode-se verificar, ainda, 
que $\eta_{r}$ é de ordem inferior a $n^{-1}$ para $r \geq 5$. Da expansão (9) encontramos, até $O\left(n^{-1}\right)$,

$$
F_{S}(x ; \kappa)=\Phi(x)-\phi(x)\left\{\frac{6 \eta_{1}+\eta_{3} h_{2}(x)}{6}+\frac{12 \eta_{2} h_{1}(x)+\eta_{4} h_{3}(x)}{24}\right\} .
$$

Identificando (23) com (15) obtemos

$$
\begin{aligned}
& A_{1}(x)=-\phi(x)\left\{6 \eta_{1}+\eta_{3} h_{2}(x)\right\} / 6 \\
& A_{2}(x)=-\phi(x)\left\{12 \eta_{2} h_{1}(x)+\eta_{4} h_{3}(x)\right\} / 24 .
\end{aligned}
$$

Assim, precisamos apenas de $b_{1}(\theta), v_{2}(\theta), \rho_{3 \hat{\theta}}$ e $\rho_{4 \hat{\theta}}$ para calcularmos a expansão da EMV até $O\left(n^{-1}\right)$ e, portanto, obtermos a EMV corrigida cuja distribuição, até esta ordem, é a normal reduzida. Aplicando a equação (17) e notando que $f_{Z}^{\prime}(S)=-S \phi(S)$, obtemos com alguma álgebra

$$
\begin{aligned}
S^{*}= & S+\frac{\left(\eta_{3}-\eta_{1}\right)}{6}+\frac{1}{72}\left\{\left(\eta_{3}-\eta_{1}\right)^{2}+9\left(\eta_{4}-4 \eta_{2}\right)\right\} S \\
& -\frac{\eta_{3}}{6} S^{2}+\frac{1}{72}\left\{2 \eta_{3}\left(\eta_{1}-\eta_{3}\right)-3 \eta_{4}\right\} S^{3}+\frac{\eta_{3}^{2}}{72} S^{5}
\end{aligned}
$$

onde $S=(\hat{\theta}-\theta) \kappa_{\theta, \theta}^{1 / 2}$. Assim, a EMV corrigida $S^{*}$ é um polinômio de $5^{\mathrm{O}}$ grau na EMV padronizada $S$.

Usamos aqui a notação $S^{*}=S+\sum_{i=0}^{5} A_{i} S^{i}$ para expressarmos a equação (24), onde

$$
\begin{aligned}
& A_{0}=\left(\eta_{3}-\eta_{1}\right) / 6, \\
& A_{1}=\left\{\left(\eta_{3}-\eta_{1}\right)^{2}+9\left(\eta_{4}-4 \eta_{2}\right)\right\} / 72, \\
& A_{2}=-\eta_{3} / 6, \\
& A_{3}=\left\{2 \eta_{3}\left(\eta_{1}-\eta_{3}\right)-3 \eta_{4}\right\} / 72, \\
& A_{4}=0 \\
& A_{5}=\eta_{3}^{2} / 72 .
\end{aligned}
$$


Mostramos abaixo como calcular os $\eta_{i}^{\prime} s$ (e, portanto, os $A_{i}^{\prime} s$ ) a partir de cumulantes de derivadas da log-verossimilhança. Precisamos das fórmulas de $b_{1}(\theta), v_{2}(\theta), \rho_{3 \hat{\theta}}$ e $\rho_{4 \hat{\theta}}$. A fórmula de $b_{1}(\theta)$ é obtida de Cordeiro e McCullagh (1991; equação (4.1)) fazendo todos os índices iguais a $\theta$. As fórmulas para $v_{2}(\theta), \rho_{3 \hat{\theta}}$ e $\rho_{4 \hat{\theta}}$ são dadas (em diferente notação) por Shenton e Bowman (1977; fórmulas (2.30b), (2.31a) e (2.31b)). Com algum algebrismo, podemos encontrar as seguintes fórmulas usando cumulantes de derivadas da logverossimilhança:

$$
\begin{aligned}
b_{1}(\theta) & =\left(\kappa_{\theta \theta}^{(\theta)}-\frac{1}{2} \kappa_{\theta \theta \theta}\right) / \kappa_{\theta, \theta}^{2}, \\
v_{2}(\theta) & =\frac{\kappa_{\theta \theta, \theta \theta}-\kappa_{\theta \theta \theta}^{(\theta)}+2 \kappa_{\theta \theta}^{(\theta \theta)}}{\kappa_{\theta, \theta}^{3}}+\frac{5 \kappa_{\theta, \theta}^{(\theta)^{2}}-\frac{1}{2} \kappa_{\theta \theta \theta}^{2}-2 \kappa_{\theta \theta}^{(\theta)} \kappa_{\theta \theta \theta}}{\kappa_{\theta, \theta}^{4}} \\
\rho_{3 \hat{\theta}} & =\frac{3 \kappa_{\theta \theta}^{(\theta)}-\kappa_{\theta \theta \theta}}{\kappa_{\theta, \theta}^{3 / 2}} \\
\rho_{4 \hat{\theta}} & =\frac{\kappa_{\theta \theta \theta \theta}-4 \kappa_{\theta \theta \theta}^{(\theta)}+6 \kappa_{\theta \theta}^{(\theta \theta)}+3 \kappa_{\theta \theta, \theta \theta}}{\kappa_{\theta, \theta}^{2}}+\frac{12 \kappa_{\theta \theta}^{(\theta)}\left(2 \kappa_{\theta \theta}^{(\theta)}-\kappa_{\theta \theta \theta}\right)}{\kappa_{\theta, \theta}^{3}} .
\end{aligned}
$$

Substituindo as fórmulas acima nas equações dos $\eta_{i}^{\prime} s$, temos:

$$
\begin{aligned}
\eta_{1}= & \left(2 \kappa_{\theta \theta}^{(\theta)}-\kappa_{\theta \theta \theta}\right) / 2 \kappa_{\theta, \theta}^{3 / 2}, \\
\eta_{2}= & \left(\kappa_{\theta \theta, \theta \theta}-\kappa_{\theta \theta \theta}^{(\theta)}\right. \\
& \left.\quad+2 \kappa_{\theta \theta}^{(\theta \theta)}\right) / \kappa_{\theta, \theta}^{2}+\left(24 \kappa_{\theta \theta}^{(\theta) 2}-12 \kappa_{\theta \theta}^{(\theta)} \kappa_{\theta \theta \theta}-\kappa_{\theta \theta \theta}^{2}\right) / 4 \kappa_{\theta, \theta}^{3}, \\
\eta_{3}= & \left(3 \kappa_{\theta \theta}^{(\theta)}-\kappa_{\theta \theta \theta}\right) / \kappa_{\theta, \theta}^{3 / 2} \\
\eta_{1}= & \left(\kappa_{\theta \theta \theta \theta}-4 \kappa_{\theta \theta \theta}^{(\theta)}+6 \kappa_{\theta \theta}^{(\theta \theta)}+3 \kappa_{\theta \theta, \theta \theta}\right) / \kappa_{\theta, \theta}^{2} \\
& \quad+2\left(9 \kappa_{\theta \theta}^{(\theta)}-\kappa_{\theta \theta \theta}\right)\left(2 \kappa_{\theta \theta}^{(\theta)}-\kappa_{\theta \theta \theta}\right) / \kappa_{\theta, \theta}^{4},
\end{aligned}
$$

eliminando as ordens dos termos desprezados. 
Inserindo estes $\eta_{i}^{\prime} s$ nas fórmulas dos $A_{i}^{\prime} s$ para obter o polinômio (24), encontramos $\left(A_{4}=0\right)$ :

$$
\begin{aligned}
& A_{0}=\left(4 \kappa_{\theta \theta}^{(\theta)}-\kappa_{\theta \theta \theta}\right) /\left(12 \kappa_{\theta, \theta}^{3 / 2}\right), \\
& A_{1}=(\left.\kappa_{\theta \theta \theta \theta}-\kappa_{\theta \theta, \theta \theta}-2 \kappa_{\theta \theta}^{(\theta \theta)}\right) /\left(8 \kappa_{\theta, \theta}^{2}\right)+\left(109 \kappa_{\theta \theta \theta}^{2}-368 \kappa_{\theta \theta}^{(\theta)} \kappa_{\theta \theta \theta}\right. \\
&\left.\quad+448 \kappa_{\theta \theta}^{(\theta)^{2}}\right) /\left(288 \kappa_{\theta, \theta}^{3}\right), A_{2}=\left(\kappa_{\theta \theta \theta}-3 \kappa_{\theta \theta}^{(\theta)}\right) /\left(6 \kappa_{\theta, \theta}^{3 / 2}\right), \\
& A_{3}=-\left(\kappa_{\theta \theta \theta}-4 \kappa_{\theta \theta \theta}^{(\theta)}+6 \kappa_{\theta \theta}^{(\theta \theta)}+3 \kappa_{\theta \theta, \theta \theta}\right) /\left(24 \kappa_{\theta, \theta}^{2}\right) \\
&+\left(73 \kappa_{\theta \theta}^{(\theta)} \kappa_{\theta \theta \theta}-7 \kappa_{\theta \theta \theta}^{2}-120 \kappa_{\theta \theta}^{(\theta)^{2}}\right) /\left(72 \kappa_{\theta, \theta}^{3}\right), \\
& A_{5}=\left(\kappa_{\theta \theta \theta}-3 \kappa_{\theta \theta}^{(\theta)}\right)^{2} /\left(72 \kappa_{\theta, \theta}^{3}\right) .
\end{aligned}
$$

Uma aplicação importante da equação (24) diz respeito ao cálculo da EMV corrigida para qualquer modelo econométrico de um único parânetro.

\section{Conclusão.}

Demonstramos que qualquer estatística satisfazendo condições gerais de regularidade, pode ser aperfeiçoada através de simples ajustamento multiplicativo para concordar com uma distribuição arbitrária de referência até $O\left(n^{-1}\right)$. O ajustamento requer $\odot$ conhecimento de expansão do tipo-Edgeworth da distribuição da estatística original até $O\left(n^{-1}\right)$. As condições que validam a estatística aperfeiçoada são as mesmas que garantem a validade da expansão da distribuição da estatística original. Na prática, o fator de correção envolve algumas derivadas da distribuição aproximada de referência, certas diferenças entre cumulantes (de ordens apropriadas em $n$ ) da estatística não-modificada e daquela variável aleatória usada como primeira aproximação e da própria estatística original. Este artigo, em nosso entender, abre um leque amplo de pesquisas em aperfeiçoamento de vários testes econométricos. 


\section{Agradecimentos.}

Os autores expressann a sua gratidão a Francisco Cribari Neto (Southern Illinois University), aos editores da Revista de Econometria e a um parecerista anônimo, pelas inúmeras sugestões que melhoraram este artigo.

Submetido em. Julho de 1995. Revisado em Outubro de 1995.

\section{Referências}

Baringhauss, L. e Henze, N. 1992. "A goodness of fit test for the Poisson distribution based on the empirical probability generating function." Statistics und Probability Let.ters 13: 269-274.

Chandra, T.K. 1985. "Asymptotic expansions of perturbed chisquare variables." Sankihyã A 47: 100--110.

Chesher. A. e Spady; R.S. 1991. "Asymptotic expansions of the information matrix test statistic." Econometrica 59: 787-815.

Cordeiro, G.M. 1993. "Bartlett corrections and bias correction for two heteroscedastic regression moclels." Communications in Statistics -- Theory and Methods 22: 169-188.

Cordciro, G.M. 1995. "Performance of a Bartlett-type modification for the deviance." J. Stutist. Comput. Simul. 51: 585--403.

Cordciro, G.M. e Ferrari, S.L. cle P. 1991. "A modified score statistic having chi-squared clistribution to oreler $n^{-1}$." Biometrika 78 : 573-582.

Cordeiro, G.M., Ferrari, S.L. de P. e Paula, G.A. 1993. "Improved score tests for generalized linear models." Journal of the Royal Statistical Societ.y B 55: 661-674.

Cordeiro, G.M. e McCullagh, P. 1991. "Bias correction in generalized linear models." Journal of the Royal Statistical Society $B$ 53: 629-643. 
Cordeiro, G.M. e Pérez-Abreu, V. 1995. "Bartlett corrections for V-statistics." Brazilian Journal of Probability and Statistics $\mathbf{9}$.

Cox, D.R. e Reid, N. 1987. "Approximations to noncentral distributions." Canadian Journal of Statistics 15: 105-114.

Cribari-Neto, F. 1994. "Asymptotically chi-squared econometric criteria." PhD Thesis, University of Illinois at Urbana-Champaign.

Davis, A. W. 1976. "Statistical distributions in univariate and multivariate Edgeworth populations." Biometrika 63: 661-670.

Davidson, R. e Mackinnon, J.G. 1992. "A new form of the information matrix test." Econometrica 60: 145-147.

Durbin, J. 1973. "Distribution theory for tests based on the sample distribution function." Reg. Conf. in Appl. Math. 9: SIAM.

Feller, W. 1971. An introduction to probability theory and its applications. Vol. 2. New York: Wiley.

Harris, P. 1985. "An asymptotic expansion for the null distribution of the efficient score statistic." Biometrika 72: 653-659.

Kendall, M.G. e Stuart, A. 1977. The advanced theory of Statistics. Vol. 2. London: Griffin.

Orme, C. 1990. "The small-sample performance of the information matrix test." Journal of Econometrics 46: 309-331.

Shenton, L.R. e Bowman, K.O. 1977. Maximum likelihood estimation in small samples. London: Griffin.

Skovgaard, I.M. 1981a. "Transformation of an Edgeworth expansion by a sequence of smooth functions." Scandinavian. Journal of Statistics 8: 207-217.

Skovgaard, I.M. 1981b. "Edgeworth expansions of the distributions of maximum likelihood estimators in the general (non i.i.d.) case." Scandinavian Journal of Statistics 8: 227-236.

Skovgaard, I.M. 1986. "On multivariate Edgeworth expansions." International Statistical Review, 54: 169-186. 
Metodologia para corrigir distribuiçōes de estatisticas

Taylor, L.W. 1987. "The size bias of White's informations matrix test." Econometrics Letters 24: 63-67.

Watson, G.S. 1961. "Goodness of fit tests on a circle." Biometrika. 48: 109-114. 